\title{
Research and Application of High Precision Machining Technology for Super- Large Integral Frame Parts of Aluminum Alloy
}

\author{
Hong $\mathrm{JI}^{1}$, Yujie WANG, Yu PENG, Lixin ZHAO and Song HUANG \\ CAC Chengdu Aircraft Industrial (Group) Co., Ltd., Chengdu 610092, China
}

\begin{abstract}
Compared with ordinary large-scale structural parts, super-large aircraft aluminum alloy integral frame parts have the characteristics of large size, high ribs and thin-walled, which lead to the difficulty of deformation control and dimensional accuracy assurance in the machining process, and the problems of spring knife and broach are easy to occur. In this paper, the research on super-large aluminum alloy integral frame parts is carried out, and a set of methods with part deformation control and coordinate drift error control are proposed, and the processing programming strategy is further optimized. This method has been successfully applied to a superlarge aircraft aluminum alloy integral frame part, which greatly reduces the deformation of parts, improves the processing stability, and improves the processing efficiency by about $30 \%$.
\end{abstract}

Keywords. super-large integral frame part, deformation control, drift error reduction, thin-walled high rib

\section{Introduction}

With the rapid upgrading of modern aircraft and the continuous improvement of aircraft performance, more and more integral frame parts are used in aircraft, and the development trend is towards super-large and complex structure. Figure 1 and Figure 2 shows a large integral frame part of Eurofighter Typhoon and a wing rib of Airbus plane, respectively [1].

The size of super-large aluminum alloy integral frame is generally larger than 3000 * $1500 * 150 \mathrm{~mm}$. The minimum thin-walled thickness is $1.5 \mathrm{~mm}$. To achieve high precision and high efficiency machining of this kind of parts is one of the difficult problems to be solved in the field of NC machining [2].

Zhang et al. have put forward effective vibration method in deformation control technology, which can solve the deformation problem of small and medium-sized wall plate thin-walled parts [3]. Dong and Cheng and others put forward a finite element correction scheme for parts deformation correction, which can predict the deformation trend of specific parts in advance and adjust accordingly [4]. However, the above

${ }^{1}$ Corresponding Author: Hong JI, CAC Chengdu Aircraft Industrial (Group) Co., Ltd., Chengdu China; 
methods cannot solve the deformation problems such as side bending, warping and shrinkage existing in the machining of super-large aluminium alloy integral frame, and cannot effectively guarantee the machining accuracy of super-large aluminium alloy integral frame. Therefore, this paper takes a super-large aluminium alloy integral frame as the application object, and puts forward a new process method which integrates deformation control, drift error control and machining strategy optimization, which can improve the deformation control ability and precision guarantee ability of super-large aluminium alloy integral frame.

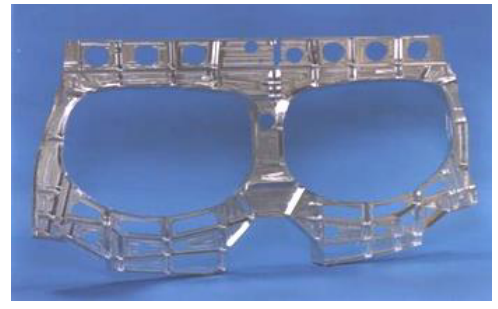

Figure 1. A large integral frame part of Eurofighter Typhoon.

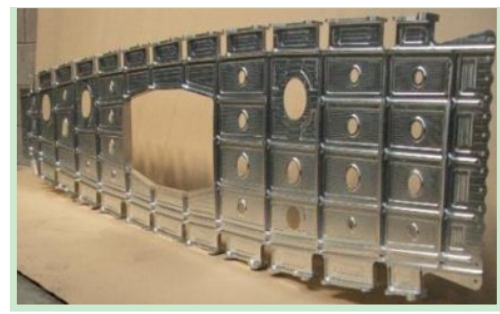

Figure 2. A wing rib of Airbus plane (15V2).

\section{Analysis of Machining Difficulties}

At present, the main problems existing in super-large aluminium alloy integral frame are as follows:

\subsection{Difficulties of Deformation Control}

The major influential factors leading to processing deformation are summarized as:

- Super large size and asymmetric structure.

- Unevenly release of residual stress and internal stress caused by large amount of material removal in the stage of rough machining.

The typical deformation trend of large-scale integrated structural component in machining is illustrated as follows (as shown in Figure 3):

- $\quad$ Lateral bending along the length with deformation of about $0.4 \mathrm{~mm}$;

- Warping along the thickness with deformation of about $3 \mathrm{~mm}$;

- Shrinking along the length with deformation of about $0.4 \mathrm{~mm}$.

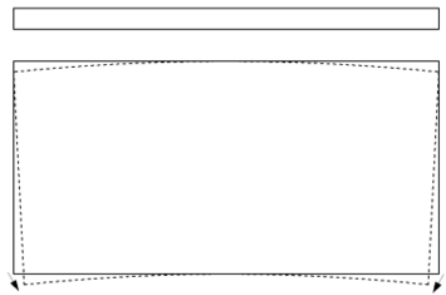

(a)

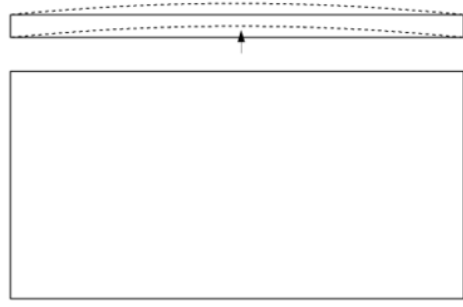

(b) 


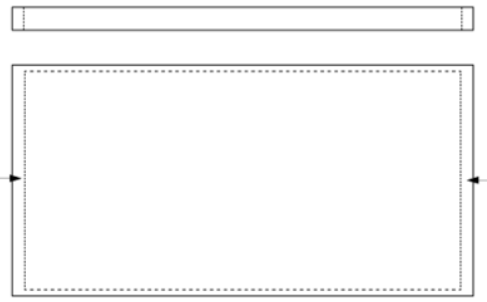

(c)

Figure 3. Schematic diagram of typical deformation trend for large integrated frame part machining: (a) lateral bending; (b) warping; (c) shrinking.

\subsection{Difficulties of Drift Error Control}

The coordinate drift exists in all axes of large-scale CNC machine tools. The maximum stroke of the coordinate axes of the machine tools is about $4000 \mathrm{~mm}$ when machining. Statistics of the coordinate axes drift of large-scale CNC machine tools are usually about $0.2-0.4 \mathrm{~mm}$ by field data collection, which seriously affects the accuracy of parts and the quality of products [5].

\subsection{Poor Stability of Machining Process}

Generally, the external profile of super-large integral frame part is designed with 1.5 2.5 $\mathrm{mm}$ ribs along outer profile, accompanied by asymmetric structure, such as the depth of cavity on one side approaching $70 \mathrm{~mm}$, that on other side reaching $95 \mathrm{~mm}$. On strong consideration for light weighting, web plates and hig-ribs are empirically designed as 1.5 $\mathrm{mm}$, except for the key areas where are susceptible to fatigue damage. In addition, the lug for mounting flange and some lightening holes are also used to saving in weight. In the high-speed milling process, such structures, like thin wall and high rib (as shown in Figure 4), make it easy to rebound or scrape the cutter. Because cutting force on the cutter is complicated, especially when it simultaneously contacts with three sides, the bottom and the lateral of lug boss as well as the web plate [6].
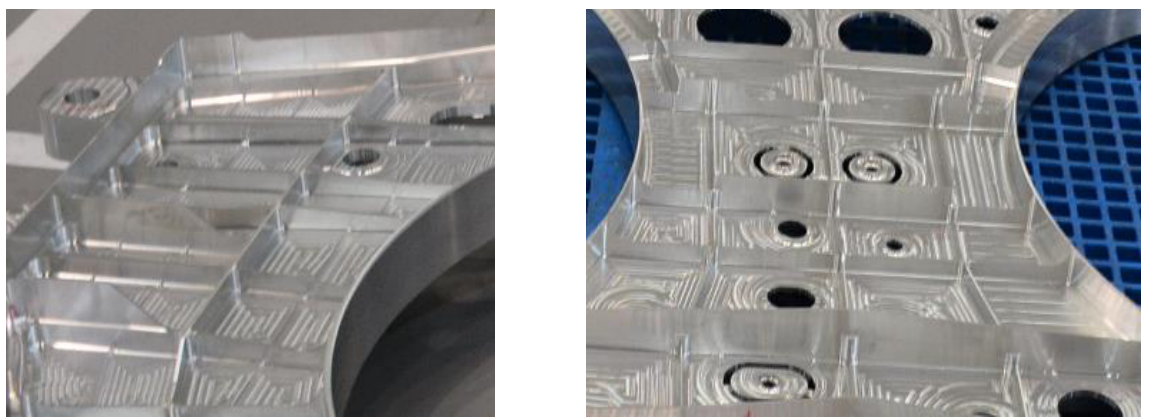

Figure 4. Typical structure of high ribs and thin web plates. 


\subsection{Long Period of Machining Process}

A typical super-large aluminum alloy integral part weighs about $3500 \mathrm{~kg}$. After rough machining, the weight of the part is about $500 \mathrm{~kg}$, the amount of blank removed from rough machining exceeds $3000 \mathrm{~kg}$, and the material removal rate is about $85 \%$. The manufacturing cycle of the whole part is 21 days. It can be imagined that such a high material removal rate and such a long-time span will pose a serious challenge to the deformation control of the part [7].

\section{High Efficiency and High Precision Strategy}

Based on the analysis of machining difficulties and production experiences on large integral frame parts, present work investigated the CNC technology with high machining precision from two aspects: distortion control and product quality control.

\subsection{Multi-Dimensional Deformation Control by Improving Process Scheme}

Technology of distortion control proposed for super large integrated frame parts is shown in Figure 5.

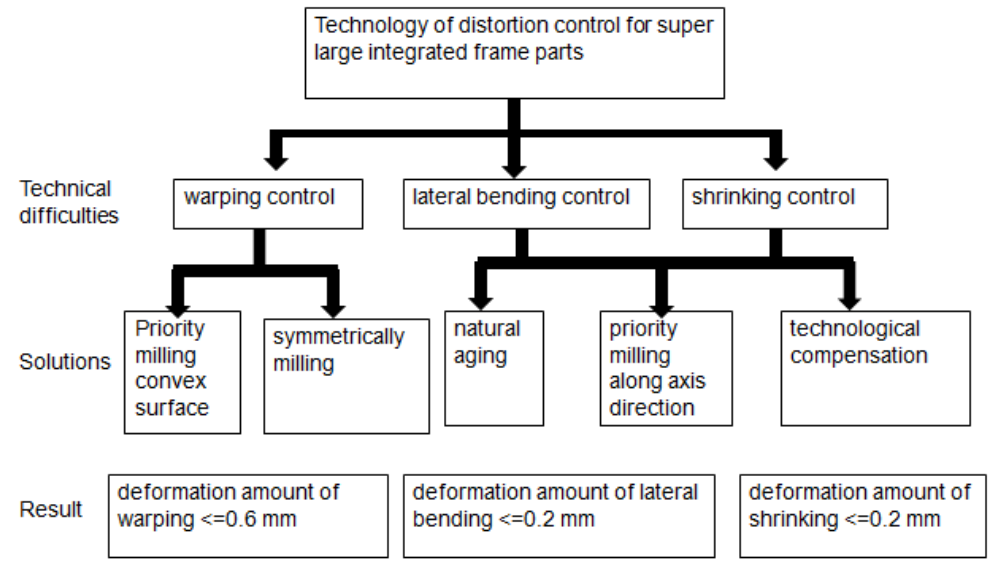

Figure 5. Technology roadmap of high-precision machining on large integral frame parts.

3.1.1. Priority milling convex surface and symmetrically machining to reduce warping Increase natural aging, combined with axial priority process compensation to reduce side bending

Forging piece as mentioned in section 2.4 (size of $3800 \times 1800 \times 180 \mathrm{~mm}$ ) itself has warping deformation of $10 \mathrm{~mm}$, but that can be even worse in case of improper distortion control during machining. In order to overcome this problem, present study proposed a method illustrated in Figure 6: first mill the convex surface on workblank that caused by itself warping after forging, and the milling depth is half of the forging blank. Warping deformation occurring in machining opposites to that from itself through all processing steps. As a result, the amount of warping deformation of final product can be reduced. 


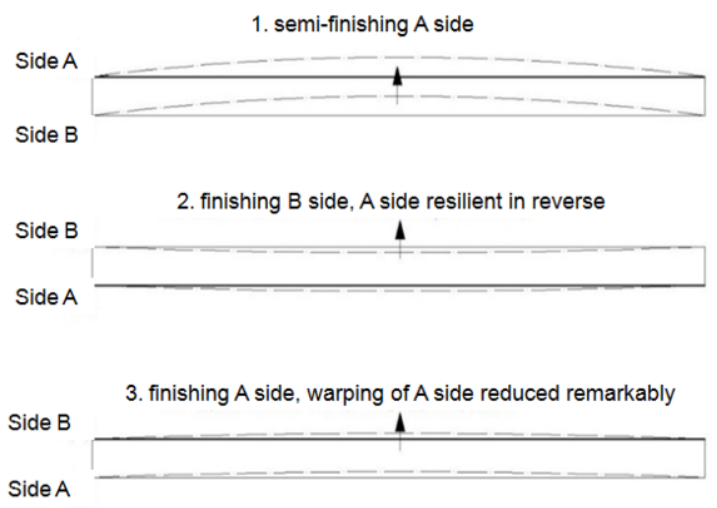

Figure 6. Method of symmetrically machining to reduce warping deformation.

\subsubsection{Increase natural aging, combined with axial priority process compensation to reduce side bending}

Due to the huge amount of material removal (sometimes even reach to 97\%) in the CNC operation of large aluminum alloy integral frame, lateral bending and shrinking often happen resulting from the effect of residual stress and cutting force on material. Generally, residual stress releases constantly as the process proceeds; then larger material removal, more distortion in parts. Thus, some approaches, such as priority milling along axis direction and natural aging, should be adopted to assist the release of residual stress.

However, machining distortions are so complicated that cannot be eliminated. According to the actual data collected about lateral bending and shrinking, aluminum alloy component with size of $1000 \mathrm{~mm}$ undergoes undesirable deformation of around 0.1 $\mathrm{mm}$ during machining. For the aluminum alloy integral frame part with profile of $3600 \times 1600 \times 165 \mathrm{~mm}$, it can be up to $0.4 \mathrm{~mm}$ that much larger than the tolerance requirement. To solve these problems, the method of technological compensation (as shown in Figure 7) is often used in practice. It can help not only minimize the distortion during milling, but also homogenize deformation in the frame parts.

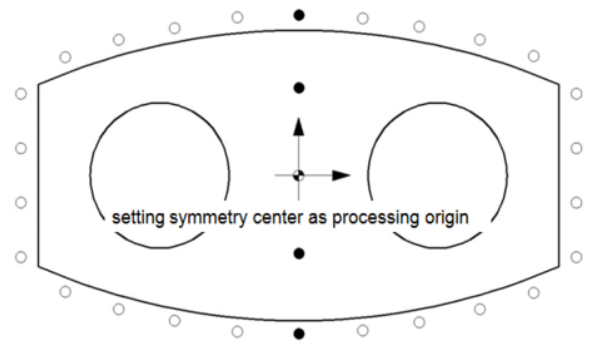

Figure 7. The origin is set at the center line of symmetry.

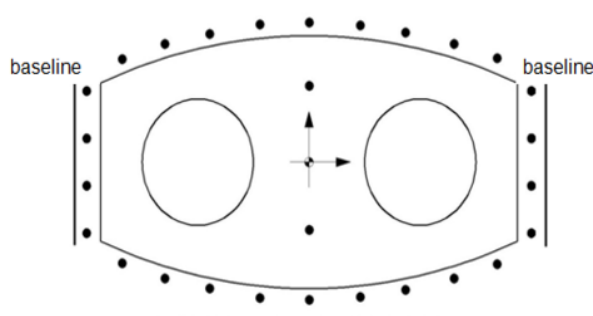

Figure 8. Set baseline. 


\subsection{Drift Error Distribution and Calibration}

Statistics show that, the drift error along $\mathrm{X}$-axis on large $\mathrm{CNC}$ machine is around 0.05 $\mathrm{mm}$ at intervals of $1000 \mathrm{~mm}$, and that on aged CNC machine is about $0.1 \mathrm{~mm}$. Through setting the symmetry center as the origin for processing, it can be reduced to $(0.1$ $0.1 / 2 / 2=) 0.075 \mathrm{~mm}$. In addition, with the help of baseline on both sides of workpiece (Figure 8), the drift error along $\mathrm{X}$-axis can be further decreased to $0.05 \mathrm{~mm}$. Based on this method, the drift error from machining large integral structure components of aluminum alloy can be smaller than $0.1 \mathrm{~mm}$.

\subsection{Optimization of Dynamic Rigid Machining Strategy for Thin-Walled High-Ribs} Strip

1. Rough machining

The material removal rate of rough machining is about $85 \%$, which is the main factor of long manufacturing cycle. Therefore, more efficient cutting parameters should be used in rough machining. The cutting parameters before and after optimization are shown in the table 1 .

Table 1. Rough machining parameters of previous and optimized program.

\begin{tabular}{cccccc}
\hline Roughing & Cutter & Speed (S) & Feed (F) & $\begin{array}{c}\text { Depth of cut } \\
\text { (AP) }\end{array}$ & $\begin{array}{c}\text { Width of cut } \\
\text { (AE) }\end{array}$ \\
\hline Previous program & D32*65R3 & 9000 & 4500 & $5 \mathrm{~mm}$ & $24 \mathrm{~mm}$ \\
\hline Optimized program & D32*65R3 & 9000 & 4500 & $5 \mathrm{~mm}$ & $18 \mathrm{~mm}$ \\
\hline
\end{tabular}

\section{Semi finishing}

In this case, high-ribs was divided into two parts for processing. During the process, the cutting depth was changed layer by layer. Finally, the upper of ribs were tapered as shown in Figure 9.
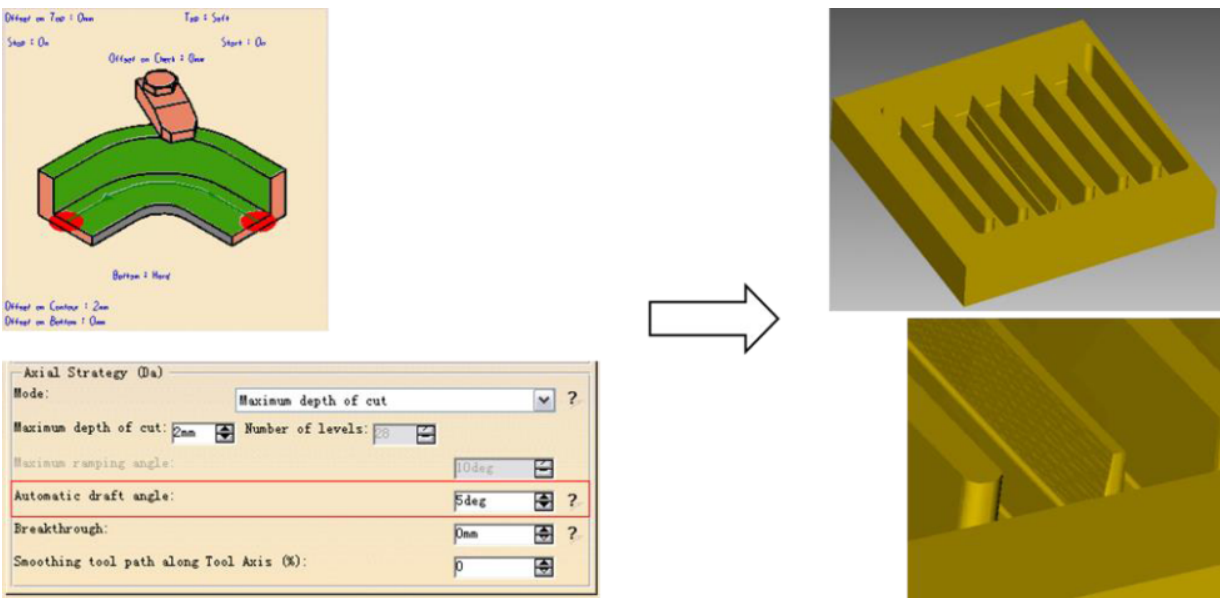

Figure 9. Semi-finishing approach: layer-by-layer machining taper. 
3. Finishing

As shown in Figure 10, the finishing process of upper part was divided into two steps. In this approach motion, spiral machining method was applied for the first part due to its poorer stiffness. But the conventional method that is single-side layer-by-layer machining, was used at the second step, because the lower part has greater stiffness and processing stability.
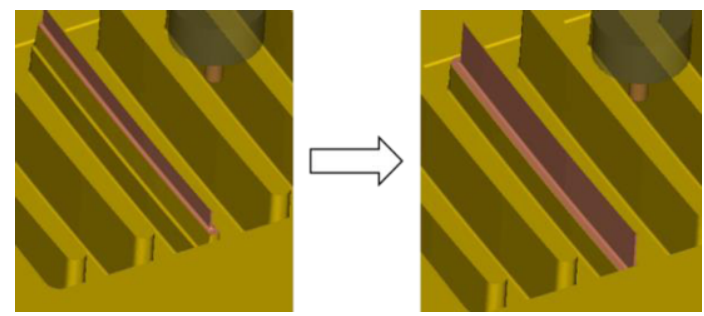

Figure 10. Finish machining approach motion: dividing finishing process for the upper part into two steps.

\section{Process Validation and Application Evaluation}

Above machining strategy was applied to a representative super-large integrated frame part of aluminum alloy. The results show that above methods possess certain processing accuracy and can be adopted in similar parts.

\subsection{Results of Distortion Control}

According to Figure 11, the deformation amount of the final product is sampled. The test results (Figure 12) indicate that distortion caused by residual stress and inhomogeneous machining stress has been controlled well and the final product is qualified. It can be concluded that the machining strategy proposed in this article can effectively solve the distortion problem in super large integrated frame parts of aluminum alloy.

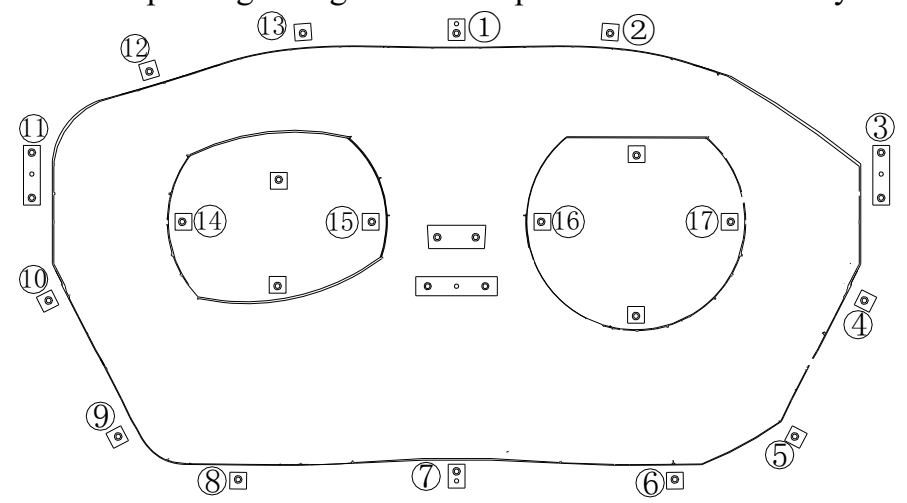

Figure 11. Sampling points used for collecting data of distortion deformation. 


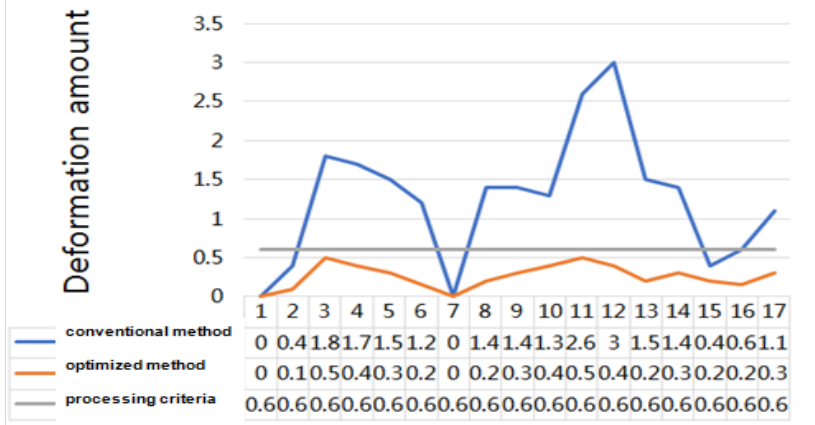

Figure 12. Comparison of deformation amount between previous and optimized program.

\subsection{Results of Surface Quality Control}

According to requirements for large structural components, surface roughness in key area for preventing fatigue damage is 1.6 , that of other areas is 3.2; dimensional tolerance of ribs, grooves and web plates is $\pm 0.2 \mathrm{~mm}$. Data from the actual measurements indicates that, after applying above machining strategy in the frame part, machining precision and surface quality were all qualified. As shown in Figure 13, there no evident trail from cutter rebounding and scraping is observed.

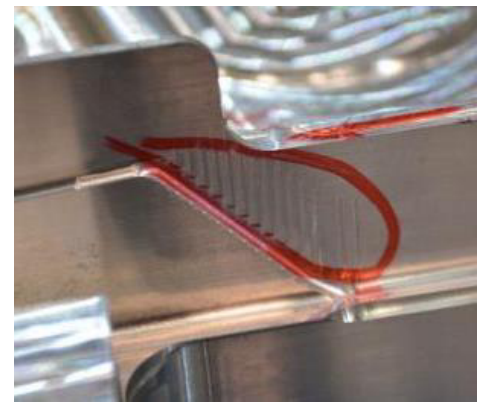

(a)

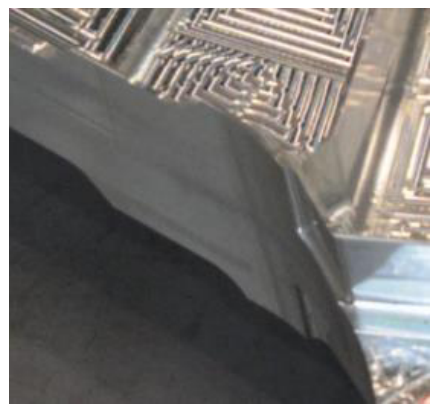

(b)

Figure 13. Surface quality in ribs (a) before and (b) after optimization.

\subsection{Results of Efficiency Improvement}

Statistics for actual processing time show that, it usually took about 21 days to finish a large integral frame part before. But, nowadays, the whole process only took 15 days by using efficiency strategy mentioned above. The processing efficiency for each product has increased by $30 \%$. In combination with the comparison of processing time in every operation between conventional method and optimized method (Figure 14), it can be concluded that the methods of efficiency improvement in present work is useful and can be implemented in super large integral frame parts. 


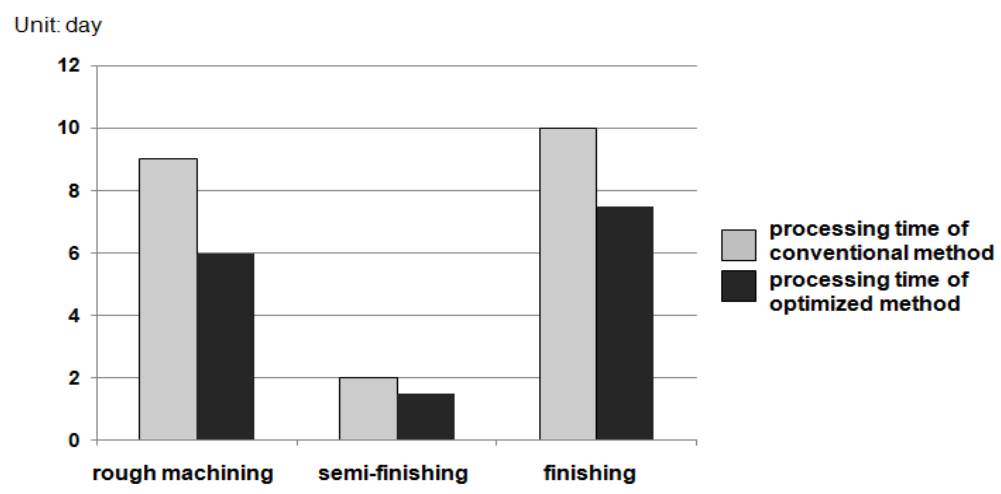

Figure 14. Processing time before and after optimization.

\section{Conclusions}

The machining strategy for representative super-large integrated frame parts of aluminum alloy was investigated. Based on analysis of technical difficulties in such parts, this study discussed the factors affecting machining deformation and dimension accuracy, proposed key technological solutions for deformation control and quality control. These machining methods were successfully implemented and promoted in NC factory of AVIC CAC. Finally, through the mass production, the qualified rate reached $99.5 \%$, and the processing efficiency increased by about $30 \%$. In summary, technological solutions of this paper are applicable to similar parts or structures with same technological characteristics. Studies in present work lay a solid foundation for super large integral frame part in aviation and accelerate the development of aviation manufacturing technology.

\section{References}

[1] Yu FF, Du BR, Ren WJ et al. Slicing recognition of aircraft integral panel generalized pocket. Chin. J. Aeronautics. 2008, 21(6):585-592.

[2] Lasemi A, Xue DY, Gu PH. Recent development in CNC machining of freeform surfaces: A state-of-theart review. Computer-Aided Design. 2010, 42(7):641-654.

[3] Zhang DJ, Cui ZS, Ruan XY, et al. An analytical model for predicting springback and side wall curl of sheet after U-bending. Comp. Mater. Sci. 2007, 38(4):707-715

[4] Dong ZC, Cheng HB. Study on removal mechanism and removal characters for SiC and fused silica by fixed abrasive diamond pellets. Int. J. Machine Tools Manuf. 2014, 85:1-13.

[5] Michael G, Josef M, Nils F et al. High precision grey-box model for compensation of thermal errors on five-axis machines. CIRP Annals - Manufacturing Tech. 2014, 63(1):509-512

[6] Masaomi T, Shintaro T, Noriyuki K et al. Enhancement of geometric accuracy of five-axis machining centers based on identification and compensation of geometric deviations. Int. J. Machine Tools Manuf. 2013, 68:11-20.

[7] Guo Q, Zhao B, Jiang Y, et al. Cutting force modeling for non-uniform helix tools based on compensated chip thickness in five-axis flank milling process. Precision Eng. 2018, 51:659-681. 